\title{
Discovery of the Young, Energetic Radio Pulsar PSR J1105-6107
}

\author{
V. M. Kaspi ${ }^{1}$ \\ Massachusetts Institute of Technology, Physics Department, Center for Space Research \\ 37-621, 70 Vassar Street, Cambridge, MA, 02139 \\ M. Bailes \\ University of Melbourne, School of Physics, Parkville, Victoria 3052, Australia \\ R. N. Manchester \\ Australia Telescope National Facility, CSIRO, PO Box 76, Epping NSW 2121, Australia \\ B. W. Stappers \\ Mount Stromlo and Siding Spring Observatories, ANU, Private Bag, Weston Creek, ACT \\ 2611, Australia \\ J. S. Sandhu \\ California Institute of Technology, MS 105-24, Pasadena, CA 91125 \\ J. Navarro ${ }^{2}$ \\ National Radio Astronomy Observatory, P.O. Box 0, Socorro, NM 87801 \\ N. D'Amico \\ Osservatorio Astronomico, via Zamboni 33, 40126 Bologna and Istituto di \\ Radioastronomia, Via P. Gobetti 101, 40129 Bologna, Italy
}

\begin{abstract}
We report the discovery and follow-up timing observations of the 63 ms radio pulsar, PSR J1105-6107. The pulsar is young, having a characteristic age of only $63 \mathrm{kyr}$ and, from its dispersion measure, is estimated to be at a distance of $\sim 7 \mathrm{kpc}$ from the Sun. We consider its possible association with the nearby supernova remnant G290.1-0.8 (MSH 11-61A); an association requires that the pulsar's proper motion be $\sim 22$ mas $\mathrm{yr}^{-1}$ (corresponding to $\sim 650 \mathrm{~km} \mathrm{~s}^{-1}$ for a distance of $7 \mathrm{kpc}$ ) directed away from the remnant center, assuming that the characteristic age is the true age. The pulsar's spin-down luminosity, $2.5 \times 10^{36} \mathrm{erg} \mathrm{s}^{-1}$, is in the
\end{abstract}

\footnotetext{
${ }^{1}$ Hubble Fellow

${ }^{2}$ Current address: Kvednaberget 12, 4033 Forus, Norway
} 
top $1 \%$ of all known pulsar spin-down luminosities. Given its estimated distance, PSR J1105-6107 is therefore likely to be observable at high energies. Indeed, it is coincident with the known CGRO/EGRET source 2EG J1103-6106; we consider the possible association and conclude that it is likely.

Subject headings: stars: neutron, pulsars: individual: (PSR J1105-6107), supernova remnants, gamma rays: observations

\section{Introduction}

We have discovered a $63 \mathrm{~ms}$ radio pulsar, PSR J1105-6107, during a recent search for pulsars using the $64 \mathrm{~m}$ radio telescope at Parkes, NSW, Australia. Most pulsars with similar rotation periods fall into one of two categories: those that are young and energetic, the short spin period a result of a relatively recent birth, or those that have been mildly recycled by a binary companion, like the original binary pulsar, PSR B1913+16, which has a spin period of $59 \mathrm{~ms}$. Thus, a $63 \mathrm{~ms}$ radio pulsar is an important find, worthy of further investigation.

Here we report on the discovery and follow-up timing observations of PSR J1105-6107, and show that it is a member of the first category above, namely young and energetic. Pulsars are hypothesized to have been born in supernovae; the existence of a young pulsar therefore requires us to consider whether there is an associated remnant of a supernova explosion. In the case of PSR J1105-6107, we consider its possible association with the known remnant G290.1-0.8 (MSH 11-61A) which lies nearby on the sky. In addition, the existence of an energetic pulsar requires us to consider whether there should be associated high-energy emission, since magnetospheric X-ray and $\gamma$-ray emission, generally representing a significant fraction of the pulsar's spin-down luminosity, very likely holds clues to the yet-elusive pulsar emission mechanism. We show that PSR J1105-6107 should be detectable at high energies, and consider whether the known EGRET $\gamma$-ray source 2EG J1103-6106 is associated with the pulsar.

\section{Observations and Results}

PSR J1105-6107 was discovered with the Parkes telescope in 1994 July in a search at

a central radio frequency of $1420 \mathrm{MHz}$. The search targeted OB runaway stars in the hope of detecting new pulsar/OB star binaries. PSR J1105-6107 was discovered while pointing at the B4V star HD96264, but the follow-up observations described below rule out any 
association. The details of the search will be described elsewhere (Kaspi, Manchester, \& D’Amico 1997).

A total of 96 timing observations of PSR J1105-6107 were obtained between 1993 July 8 and 1996 July 4 at Parkes. Of these, 91 were obtained at central radio frequencies ranging from 1390 to $2050 \mathrm{MHz}$. The remaining five observations were obtained at $660 \mathrm{MHz}$ in 1995 July. All data taken before 1995 were obtained using filter-bank timing systems $(2 \times 64 \times$ $5 \mathrm{MHz}$ at $1520 \mathrm{MHz}$ and $2 \times 256 \times 0.125 \mathrm{MHz}$ at $660 \mathrm{MHz}$ ) that have been described in detail elsewhere (e.g. Bailes et al. 1994). Most data from after 1995 were obtained using the Caltech correlator-based pulsar timing machine (Navarro 1994), which has $2 \times 128$ lags across $128 \mathrm{MHz}$ in each of two separate frequency bands. Typically, correlator observations were made at central frequencies of 1420 and $1650 \mathrm{MHz}$ simultaneously. Compared to the filterbank system, the correlator's narrower frequency channels resulted in less channel dispersion smearing and hence finer time resolution. Filter-bank data were recorded on tape and folded off-line; correlator data were folded on-line. Average profiles were convolved with high signal-to-noise ratio templates to yield pulse arrival times. The average profile at $1650 \mathrm{MHz}$ shown in Figure 1 was obtained by aligning and summing numerous individual correlator profiles. The profile at $1420 \mathrm{MHz}$ is similar, while the two components obvious in Figure 1] cannot be resolved in the $660 \mathrm{MHz}$ data because of dispersion smearing. Resulting arrival times were analyzed using the standard TEMPO pulsar timing software package (Taylor \& Weisberg 1989) together with the JPL DE200 ephemeris (Standish 1982). Typical arrival time uncertainties were $\sim 250 \mu$ s for $\sim 10$ min integrations with signal-to-noise ratio $\sim 20$ at frequencies above $1390 \mathrm{MHz}$. Arrival times at $660 \mathrm{MHz}$ had uncertainties approximately twice as large.

In the timing analysis, the pulsar dispersion measure (DM) was first determined from delays across the observed bands and then refined using 15 arrival times measured in 1995 July, including all five measured at $660 \mathrm{MHz}$. This was to ensure good frequency coverage, and no contamination from long-term timing noise so common to young pulsars. The measured DM is $(271.01 \pm 0.02) \mathrm{pc} \mathrm{cm}^{-3}$ and during the subsequent timing analysis it was held fixed at this value. To minimize contamination of the timing position from long-term timing noise, we "pre-whitened" the data (e.g. Kaspi, Taylor, \& Ryba 1994), fitting for sufficiently many frequency derivatives (four) to render the residuals approximately Gaussian distributed, determined by eye. The timing position, determined while fitting for these derivatives, is given in Table 1, and was subsequently held fixed. Finally, we measured the best period and period derivative, also given in Table 11. The uncertainties in all parameters are $1 \sigma$ statistical uncertainties, obtained assuming equal weighting for all arrival times. The pulsar's surface magnetic field $B=3.2 \times 10^{19} \mathrm{G}(P \dot{P})^{1 / 2} \simeq 1 \times 10^{12} \mathrm{G}$, and its spin-down luminosity $\dot{E}=4 \pi^{2} I \dot{P} / P^{3} \simeq 2.5 \times 10^{36} \mathrm{erg} \mathrm{s}^{-1}$, where the neutron star moment of inertia $I$ 
is taken to be $10^{45} \mathrm{~g} \mathrm{~cm}^{2}$. The pulsar's characteristic age $\tau_{c} \equiv P / 2 \dot{P} \simeq 63 \mathrm{kyr}$.

Post-fit residuals, obtained after removing the timing model given in Table 1, are shown in Figure 2. In the plot, uncertainties are typically much smaller than the size of the symbol. The timing noise, interpreted as irregularities in the neutron star's rotation, is obvious. We can quantify the amount of timing noise, as prescribed by Arzoumanian et al. (1994), by measuring $\Delta_{8} \equiv \log \left(|\ddot{\nu}| t^{3} / 6 \nu\right)$, where $\nu=1 / P$ and for $t=10^{8}$ s. For PSR J1105-6107 we find $\Delta_{8}=-0.8$, which is consistent within the scatter with $\Delta_{8}$ parameters for other young pulsars. The pulsar's small $\tau_{c}$, as well as the large amount of timing noise, suggest that PSR J1105-6107 is an excellent candidate for glitches. Indeed we cannot rule out some contamination of the timing parameters by a slowly-relaxing glitch that occurred before 1993 July (c.f. Manchester et al. 1991; Lyne et al. 1996a).

That the timing observations for PSR J1105-6107 reported here extend back a full year before the pulsar's discovery requires some explanation. In general, raw, dispersed, and unfolded filter-bank timing data are recorded and archived on tape. By chance, PSR J1105-6107 lies less than one Parkes $1420 \mathrm{MHz}$ primary beam-width from the pulsar PSR J1103-6101, which was discovered in 1992 July, as part of a major search for pulsars near supernova remnants (Kaspi et al. 1996). The DM toward PSR J1103-6101 is only 75 pc $\mathrm{cm}^{-3}$, indicating that it is a foreground object. Once the discovery of PSR J1105-6107 was made and its proximity to PSR J1103-6101 realized, the archived raw data for the latter were retrieved, dedispersed, and folded at the former's parameters. [

\section{Discussion}

\subsection{Possible Association with G290.1-0.8}

PSR J1105-6107 is located near the Galactic supernova remnant G290.1-0.8, also known as MSH 11-61A (Shaver \& Goss 1970). The proximity of a young pulsar to a supernova remnant suggests that they may have been formed in the same explosion. Alternatively they may be coincidentally superposed on the sky; the Galactic plane is replete with pulsars and supernova remnants and the possibility of chance alignment is non-negligible. Indeed a spurious association is not implausible, as the true remnant of the pulsar's birth may

\footnotetext{
${ }^{3}$ PSR J1105-6107 was not detected as part of the Kaspi et al. (1996) search because it was outside the Parkes search beam. Only in 1993 July was the correct timing position for PSR J1103-6101 determined. Observations made after that date had the telescope pointing at PSR J1103-6101's refined position, which was closer to that of PSR J1105-6107.
} 
well have faded from view (Braun, Goss \& Lyne 1989), and the explosion that produced G290.1-0.8 may not have produced a neutron star. Here we consider whether there is a genuine association between PSR J1105-6107 and G290.1-0.8.

The Taylor \& Cordes (1993) DM-distance model places PSR J1105-6107 at a distance of $7 \mathrm{kpc}$, given its DM and Galactic coordinates. The uncertainty on this distance is estimated to be $\sim 25 \%$. Thus, the range consistent with the DM-distance model is $5-9 \mathrm{kpc}$. Regions of enhanced free-electron density (like H II regions) along the line-of-sight can result in an overestimate of the pulsar's distance from its DM.

The distance to G290.1-0.8 has been estimated many times in the literature. First, HI absorption measurements made by Dickel (1973) suggest that the remnant is probably at 3-4 kpc. The $\Sigma-D$ relation, which is known to be very uncertain, suggests a distance of 3-6 kpc (Clark \& Caswell 1976). Other authors have suggested that the remnant is more distant $(12-14 \mathrm{kpc})$, on the basis of its optical morphology and $\mathrm{H} \alpha$ to $[\mathrm{S} \mathrm{II}]$ ratio, which are more typical of older (hence larger) remnants like the Monoceros Ring (Elliott \& Malin 1979; Kirshner \& Winkler 1979). More recently, Rho (1995) concluded from the neutral hydrogen absorption component of the remnant's X-ray spectrum (see below) that the distance is $\sim 7 \mathrm{kpc}$, consistent with the pulsar distance, although this method has large uncertainties. Independently, Rosado et al. (1997) argued that the remnant must be at $\sim 7 \mathrm{kpc}$, on the basis of the kinematics of the optical emission, although they too concede that the estimate is uncertain given the complexity of the field. Thus, overall, the estimated distances to the remnant are generally consistent with that of the pulsar within the substantial uncertainties; an association is therefore plausible.

The age $\tau$ of a radio pulsar is given by

$$
\tau=\frac{P}{(n-1) \dot{P}}\left[1-\left(\frac{P_{0}}{P}\right)^{n-1}\right],
$$

where $P$ is its current spin period, $P_{0}$ is its spin period at birth, and $n$ is its braking index. The braking index $n$ is defined by the pulsar spin-down $\dot{\nu}=-K \nu^{n}$, where $\nu \equiv 1 / P$, and $K$ is a positive constant that depends on the magnetic dipole moment and moment of inertia of the rotating neutron star (Manchester \& Taylor 1977). It is easy to show that $n=\nu \ddot{\nu} / \dot{\nu}^{2}$, and hence can be determined from timing observations in the absence of strong timing noise. The characteristic age is defined as $\tau_{c} \equiv P / 2 \dot{P}$, which assumes that $n=3$ (true for a simple dipole) and $P_{0}<<P$. For PSR J1105-6107, $\tau_{c}=63 \mathrm{kyr}$. However, the short spin period for PSR J1105-6107 compared with other, younger pulsars (e.g. PSR B1509-58, $\tau_{c}=1.5 \mathrm{kyr}$, $P=150 \mathrm{~ms})$ suggests that $P_{0}<<P$ does not necessarily hold in this case, and that the true age may be smaller. Alternatively, if the braking index $n<3$, as is the case for all pulsars for which it has been measured, then $\tau_{c}$ is an underestimate. Notable is the recent 
measurement by Lyne et al. (1996b) of $n=1.6 \pm 0.4$ for the Vela pulsar. Figure 3 shows how the true age of PSR J1105-6107 depends on $P_{0}$ for four values of $n$. For small $P_{0}$, the pulsar's age may be anywhere between 63 and 250 kyr. For $P_{0} \simeq P$, the pulsar's age must be smaller than $63 \mathrm{kyr}$, independent of $n$. If we assume the pulsar was born with a spin period of $\sim 20 \mathrm{~ms}$, as for the Crab pulsar, then $63<\tau<110 \mathrm{kyr}$.

Age estimates for the remnant depend strongly on its distance. Milne et al. (1989) estimated the remnant to be only $2.2 \mathrm{kyr}$ old, assuming the smallest distance estimate. Rho (1995) suggests the remnant is somewhat older, $\sim 10 \mathrm{kyr}$. The resemblance of the optical emission to that of the Monoceros Ring, whose age is $\sim 50$ kyr (Leahy, Naranan, \& Singh 1986), suggests a much larger age for G290.1-0.8. If it is associated with PSR J1105-6107, the most likely range of true pulsar ages requires the remnant to have an age significantly larger than the Milne et al. estimate, more in line with the more recently suggested hypotheses that it is at a greater distance.

Figure 6 shows the location of the pulsar with respect to the remnant. It lies just over two remnant radii from the approximate remnant geometric center. Its location, well outside the remnant boundaries, argues against an association (Gaensler \& Johnston 1995). However, for a distance of $7 \mathrm{kpc}$, and assuming the age of the system to be $63 \mathrm{kyr}$, the transverse velocity of the pulsar, if it is associated with the remnant, is $\sim 650 \mathrm{~km} \mathrm{~s}^{-1}$, larger than the mean pulsar transverse velocity (Lyne \& Lorimer 1994), but much less than has been suggested for pulsars in other proposed associations (e.g. Frail \& Kulkarni 1991; Manchester et al. 1991; Caraveo 1993), and well within the range of measured pulsar velocities (Lyne \& Lorimer 1994). Thus, that the pulsar lies well outside the remnant does not necessarily rule out an association; it would require the pulsar transverse velocity to exceed the mean remnant expansion velocity by more than a factor of two, not unreasonable if the remnant is expanding into a dense environment, as is suggested by its axisymmetric morphology. Radio maps of the region closer to the pulsar show no evidence for any emission that might suggest another, closer supernova remnant, or a bow shock nebula (A. Green, personal communication). Perhaps interestingly, the pulsar's inferred trajectory approximately bisects the remnant along its line of symmetry.

Seward (1990) and Rho (1995) presented X-ray images of G290.1-0.8 that show that the emission is centrally peaked. This is in contrast to the radio morphology which is more shelllike. This suggests that G290.1-0.8 is like the supernova remnant W44 (Rho et al. 1994), which, like W28 and 3C400.2 (Long et al. 1991), has centrally peaked X-ray emission but shell-like radio morphology. The central X-ray emission in these remnants is thermal. One possible interpretation is that the emission is due to the evaporation of dense cloudlets that survived the initial blast wave, rather than a central neutron star, as is the case for remnants 
with centrally peaked radio and non-thermal X-ray emission, such as the Crab nebula. Since the morphology suggests that this is also true of G290.1-0.8, there is no evidence for a central point source that would argue against an association with PSR J1105-6107. We note also that W44, W28, and 3C400.2 are relatively old remnants, all having estimated ages greater than $10 \mathrm{kyr}$.

A measurement of the proper motion for PSR J1105-6107 is highly desirable for determining whether it is associated with G290.1-0.8. If the association is real, the pulsar proper motion should be $\sim 22(63 \mathrm{kyr} / \tau)$ mas $\mathrm{yr}^{-1}$, independent of the distance. A timing proper motion will not be forthcoming, given the large amount of timing noise exhibited by the pulsar (Figure 2). Also, its low flux density (see Table 1) will make interferometric observations using currently available telescopes difficult, although pulse gating may improve the feasibility. A measurement of the pulsar's scintillation speed through observations of its radio dynamic spectrum, a technique recently used by Nicastro, Johnston \& Koribalski (1996) to argue against an association between the radio pulsar PSR B1706-44 and the supernova remnant G343.1-2.3, may provide some evidence against an association if a small speed is found. However, this will be difficult again because of the pulsar's low flux density. A large pulsar velocity away from the remnant could be confirmed by the presence of $\mathrm{H} \alpha$ emission from a bow shock nebula (c.f. Cordes, Romani, \& Lundgren 1993), although a lack of such emission could be due to an absence of ambient neutral hydrogen, and would not disprove an association.

\subsection{Possible association with $\gamma$-ray source 2EG J1103-6106}

At a distance of $7 \mathrm{kpc}$, given its large spin-down luminosity (Table 1), PSR J1105-6107 ranks 19 th in a list of rotation-powered pulsars ordered by $\dot{E} / d^{2}$. Six of the seven top spots are held by known $\gamma$-ray pulsars (the seventh being the millisecond pulsar PSR J0437-4715), while most of the top 30 are known X-ray sources. On this list, PSR J1105-6107 ranks 15 spots higher than the known X-ray and $\gamma$-ray pulsar PSR B1055-52 (Cheng \& Helfand 1983; Fierro et al. 1993). Thus, PSR J1105-6107 is a good candidate to be an observable high-energy emitter.

In fact, the radio timing position of PSR J1105-6107 (Table 1) lies well inside the 95\% confidence $49^{\prime} \times 32^{\prime}$ error ellipse of the second EGRET catalog source 2EG J1103-6106 (Thompson et al. 1995). This $\gamma$-ray source was referred to in the first EGRET catalog as GRO J1110-60 (Fichtel et al. 1994), and is near, but outside, the error box of the second COS-B catalog source 2CG 288-00 (Swanenburg et al. 1981). Reported $E>100 \mathrm{MeV}$

fluxes of 2EG J1103-6106 show no evidence for significant variability, consistent with its 
interpretation as a rotation-powered pulsar (Thompson et al. 1995; Ramanamurthy et al. 1995). If the sources are associated, the estimated mean flux of 2EG J1103-6106 suggests that PSR J1105-6107 converts approximately 3\% of its spin-down luminosity to high-energy $\gamma$-rays for a beaming angle of $1.0 \mathrm{sr}$, comparable with the efficiencies of the Vela pulsar and PSR B1706-44 (Thompson et al. 1992; Grenier, Hermsen, \& Clear 1988).

There are 18 radio pulsars within $10^{\circ}$ of the Galactic plane that have higher $\dot{E} / d^{2}$ than PSR B1055-52 for which pulsations have not yet been detected by EGRET, omitting millisecond pulsars. With the discovery of PSR J1105-6107, four of these lie within the 99\% confidence contours of unidentified EGRET sources. By contrast, of 268 known radio pulsars within $10^{\circ}$ of the Galactic plane whose energetics should be below the EGRET threshold for detection, only two lie within the $99 \%$ confidence contours of unidentified EGRET sources (Fierro 1995). Assuming that this control group is spatially distributed like the young pulsars, using Poisson statistics, the probability for four coincidences among the 18 energetic pulsars is $\sim 1 \times 10^{-5}$. Even conservatively accounting for the possibility that the control group is less concentrated near the Galactic plane (for example, by assigning it a significantly larger mean z-height), we find that the probability for four coincidences must be under $\sim 1 \%$, although exact probabilities are difficult to estimate given the uncertainties in pulsar distances and spatial distributions, and in unidentified EGRET source properties. Even so, the evidence argues strongly that at least three of the four coincidences of high $\dot{E} / d^{2}$ pulsars with the unidentified EGRET sources are real. Furthermore, Yadigaroglu \& Romani (1997) showed that most of the unidentified low-latitude EGRET sources such as 2EG J1103-6106 are likely to be young pulsars like PSR J1105-6107. We therefore conclude that the association between PSR J1105-6107 and 2EG J1103-6106 is likely. However, only the detection of $\gamma$-ray pulsations at the radio period will demonstrate the association unambiguously.

Several authors have argued that the $\gamma$-ray source 2EG J1103-6106, as well as GRO J1110-60 and 2CG 288-00, are associated with the Carina complex, which includes the peculiar star $\eta$ Car, open clusters $\operatorname{Tr} 16$, $\operatorname{Tr} 14$, and several OB associations, with the $\gamma$-rays being produced by cosmic ray interactions in the intercluster gas, or by $\eta$ Car itself (Morfill, Forman, \& Bignami 1984; Borgwald \& Friedlander 1993; Manchanda et al. 1996). The identification of 2EG J1103-6106 with PSR J1105-6107 does not necessarily preclude these interpretations, since 2EG J1103-6106 may be a composite of several sources. Indeed there is marginal evidence that its emission is extended (Swanenburg et al. 1981; Thompson et al. 1995). Nevertheless, the discovery of a luminous young pulsar near the $\gamma$-ray source casts some doubts on alternative interpretations. Sturner \& Dermer (1995) suggested that GRO J1110-60 is associated with the supernova remnant G291.0-0.1 (MSH 11-62), with the emission a result of cosmic ray interactions with the remnant. With the revisions made in the second EGRET 
catalog, the source is now closer to the position of PSR J1105-6107 and G290.1-0.8; this and the discovery of PSR J1105-6107 suggest that their proposed model is not relevant to this particular $\gamma$-ray source.

Kaaret \& Cottam (1996) suggested that 2EG J1103-6106 is a young pulsar associated with the $\mathrm{OB}$ association Car 2. The measured distance to the association is $2.2 \mathrm{kpc}$ (Mel'nik \& Efremov 1995), which is inconsistent with the DM-derived distance of $7 \mathrm{kpc}$ for PSR J1105-6107, suggesting that the association lies in the foreground. If the pulsar actually is in the cluster, its association with G290.1-0.8 is doubtful because the remnant dimensions would suggest that it is much younger than the pulsar, and it would be hard to understand the pulsar's position so far outside the remnant since the latter would have had less time to decelerate. In this case, the pulsar should be a bright X-ray source, with X-ray luminosity $\sim 2 \times 10^{-11} \mathrm{erg} \mathrm{s}^{-1} \mathrm{~cm}^{-2}$ (Seward \& Wang 1988), and should be easily detected by X-ray satellites such as $A S C A$.

\section{Conclusions}

We have reported the discovery and follow-up timing observations of PSR J1105-6107 which show it to be young and energetic. We have considered its proximity to the supernova remnant G290.1-0.8 and show that an association between the two is possible, and could be confirmed or disproved by proper-motion measurements. We have also considered a possible association of PSR J1105-6107 with the EGRET source 2EG J1103-6106 and conclude that it is likely.

It is remarkable that this interesting pulsar was found serendipitously in a search unrelated to either EGRET sources or supernova remnants, while recent targeted searches of both have been done but have met very limited success. (e.g. Kaspi et al. 1996; Gorham et al. 1996; Nice \& Sayer 1996). That PSR J1105-6107 was missed by a survey including G290.1-0.8 is not surprising given the pulsar's low flux density and large angular displacement from the remnant. If the association between PSR J1105-6107 and G290.1-0.8 is one day proven, it, and other plausible pulsar/SNR associations in which the pulsar lies outside the remnant boundaries (see Kaspi 1996 for a review), would argue strongly that care must be taken to search a large area around the remnant, not just inside. Either way, the discovery of PSR J1105-6107 suggests that deeper searches of the error boxes of unidentified EGRET sources for radio pulsars are warranted.

We thank Joseph Fierro for helpful discussions regarding EGRET source coincidences

with radio pulsars, Shri Kulkarni for his role in building the Caltech correlator, Anne Green 
for useful discussions, and Bryan Gaensler for help with the remnant image. The filter-bank systems used in the observations were constructed at the University of Manchester, Jodrell Bank. Part of this research was carried out at the Jet Propulsion Laboratory, California Institute of Technology, under contract with the National Aeronautics and Space Administration. VMK received support from NRAO, and from Hubble Fellowship grant number HF-1061.01-94A from the Space Telescope Science Institute, which is operated by the Association of Universities for Research in Astronomy, Inc., under NASA contract NAS5-26555. 


\section{REFERENCES}

Arzoumanian, Z., Nice, D. J., Taylor, J. H., \& Thorsett, S. E. 1994, ApJ, 422, 671

Bailes, M. et al. 1994, ApJ, 425, L41

Borgwald, J. M. \& Friedlander, M. W. 1993, ApJ, 408, 230

Braun, R., Goss, W. M., Lyne, A. G. 1989, ApJ, 340, 355

Caraveo, P. A. 1993, ApJ, 415, L111

Cheng, A. F. \& Helfand, D. J. 1983, ApJ, 271, 271

Clark, D. H. \& Caswell, J. L. 1976, MNRAS, 174, 267

Cordes, J. M., Romani, R. W., \& Lundgren, S. C. 1993, Nature, 362, 133

Dickel, J. R. 1973, Astrophys. Lett., 15, 61

Elliott, K. H. \& Malin, D. F. 1979, MNRAS, 186, 45P

Fichtel, C. E. et al. 1994, ApJS, 94, 551

Fierro, J. M. 1995. PhD thesis, Stanford University

Fierro, J. M. et al. 1993, ApJ, 413, L27

Frail, D. A. \& Kulkarni, S. R. 1991, Nature, 352, 785

Gaensler, B. M. \& Johnston, S. 1995, MNRAS, 277, 1243

Gorham, P. W., Ray, P. S., Anderson, S. B., Kulkarni, S. R., \& Prince, T. A. 1996, ApJ, 458,257

Grenier, I. A., Hermsen, W., \& Clear, J. 1988, A\&A, 204, 117

Kaaret, P. \& Cottam, J. 1996, ApJ, 462, L35

Kaspi, V. M., Taylor, J. H., \& Ryba, M. F. 1994, ApJ, 428, 713

Kaspi, V. M. 1996, IAU Coll. 160, "Pulsars: Problems and Progress," eds. S. Johnston, M. A. Walker, M. Bailes, ASP,

Kaspi, V. M., Manchester, R. N., \& D’Amico, N. 1997, in preparation 
Kaspi, V. M., Manchester, R. N., Johnston, S., Lyne, A. G., \& D'Amico, N. 1996, Astron. J., 111, 2028

Kirshner, R. P. \& Winkler, P. F. 1979, ApJ, 227, 853

Leahy, D. A., Naranan, S., \& Singh, K. P. 1986, Ap\&SS, 119, 249L

Long, K. S., Blair, W. P., Matsui, Y., \& White, R. L. 1991, ApJ, 373, 567

Lyne, A. G., Kaspi, V. M., Bailes, M., Manchester, R. N., Taylor, H., \& Arzoumanian, Z. 1996a, MNRAS, 281, L14

Lyne, A. G., Pritchard, R. S., Graham-Smith, F., \& Camilo, F. 1996b, Nature, 381, 497

Lyne, A. G. \& Lorimer, D. R. 1994, Nature, 369, 127

Manchanda, R. K. et al. 1996, A\&A, 305, 457

Manchester, R. N., Kaspi, V. M., Johnston, S., Lyne, A. G., \& D’Amico, N. 1991, MNRAS, $253,7 \mathrm{P}$

Manchester, R. N. \& Taylor, J. H. 1977, Pulsars, (San Francisco: Freeman)

Mel'nik, A. M. \& Efremov, Y. N. 1995, Sov. Astron. Lett., 21, 10

Milne, D. K., Caswell, J. L., Kesteven, M. J., Haynes, R. F., \& Roger, R. S. 1989, Pub. Astr. Soc. Aust., 8, 187

Morfill, G. E., Forman, M., \& Bignami, G. 1984, ApJ, 284, 856

Navarro, J. 1994. PhD thesis, California Institute of Technology

Nicastro, L., Johnston, S., \& Koribalski, B. 1996, A\&A, 306, 49

Nice, D. J. \& Sayer, R. W. 1996, ApJ. In press

Ramanamurthy, P. V. et al. 1995, ApJ, 450, 791

Rho, J. 1995. PhD thesis, University of Maryland

Rho, J., Petre, R., Schlegel, E., \& Hester, J. 1994, ApJ, 430, 757

Rosado, M., Ambrocio-Cruz, P., Le Coarer, E., \& Marcelin, M. 1997, A\&A. submitted

Seward, F. D. 1990, ApJS, 73, 781 
Seward, F. D. \& Wang, Z.-U. 1988, ApJ, 332, 199

Shaver, P. A. \& Goss, W. M. 1970, Aust. J. Phys. Astr. Supp., 14, 77

Standish, E. M. 1982, A\&A, 114, 297

Sturner, S. J. \& Dermer, C. D. 1995, A\&A, 293, L17

Swanenburg, B. N. et al. 1981, ApJ, 243, L69

Taylor, J. H. \& Cordes, J. M. 1993, ApJ, 411, 674

Taylor, J. H. \& Weisberg, J. M. 1989, ApJ, 345, 434

Thompson, D. J. et al. 1992, Nature, 359, 615

Thompson, D. J. et al. 1995, ApJS, 101, 259

Whiteoak, J. B. Z. \& Green, A. J. 1996, A\&AS, 118, 329

Yadigaroglu, I. A. \& Romani, R. W. 1997, ApJ. in press 


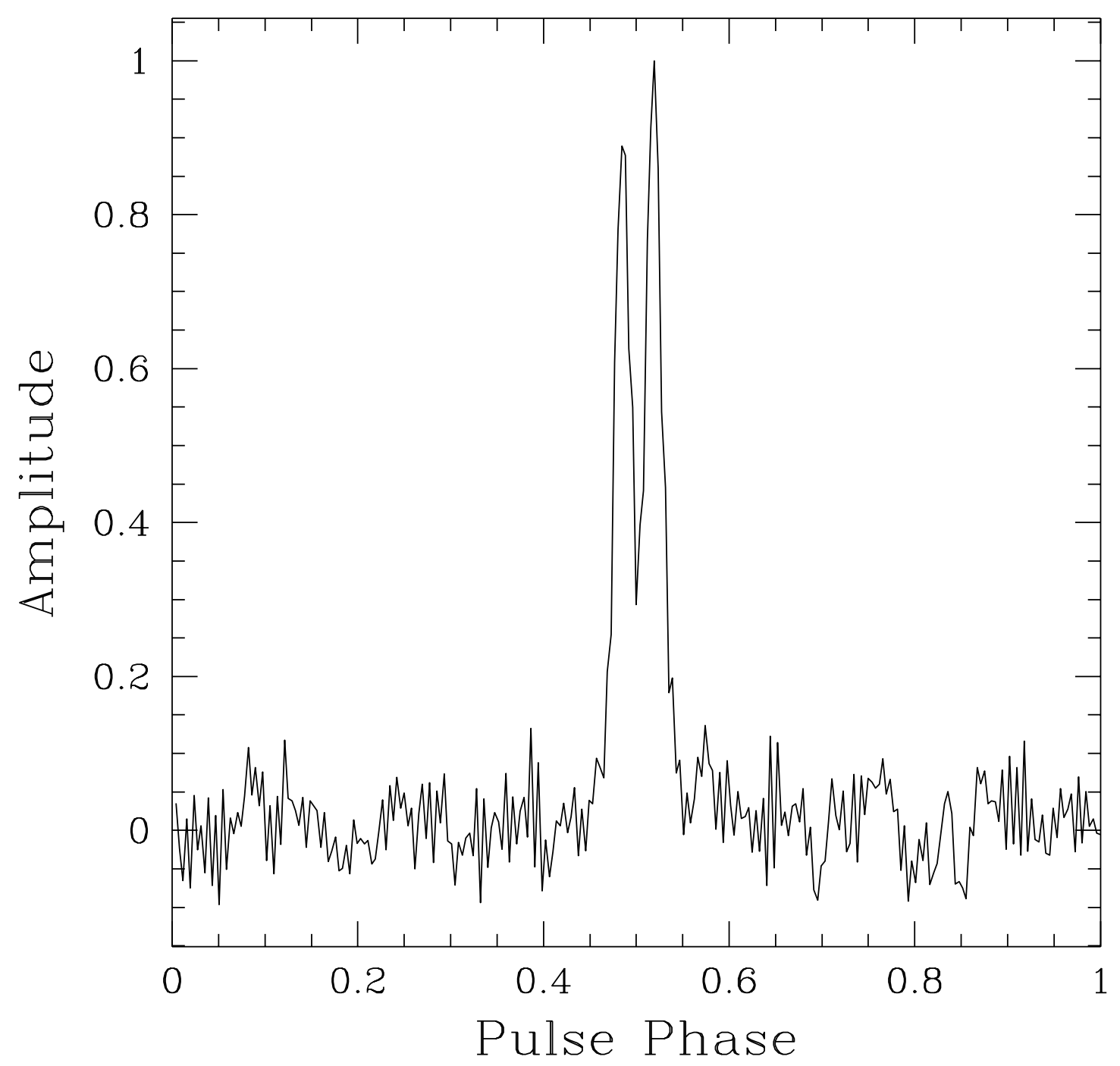

Fig. 1.- Pulse profile for PSR J1105-6107 at $1650 \mathrm{MHz}$, with peak normalized to unity. The profile is similar at $1420 \mathrm{MHz}$. The apparent off-pulse structure is a result of interference. 


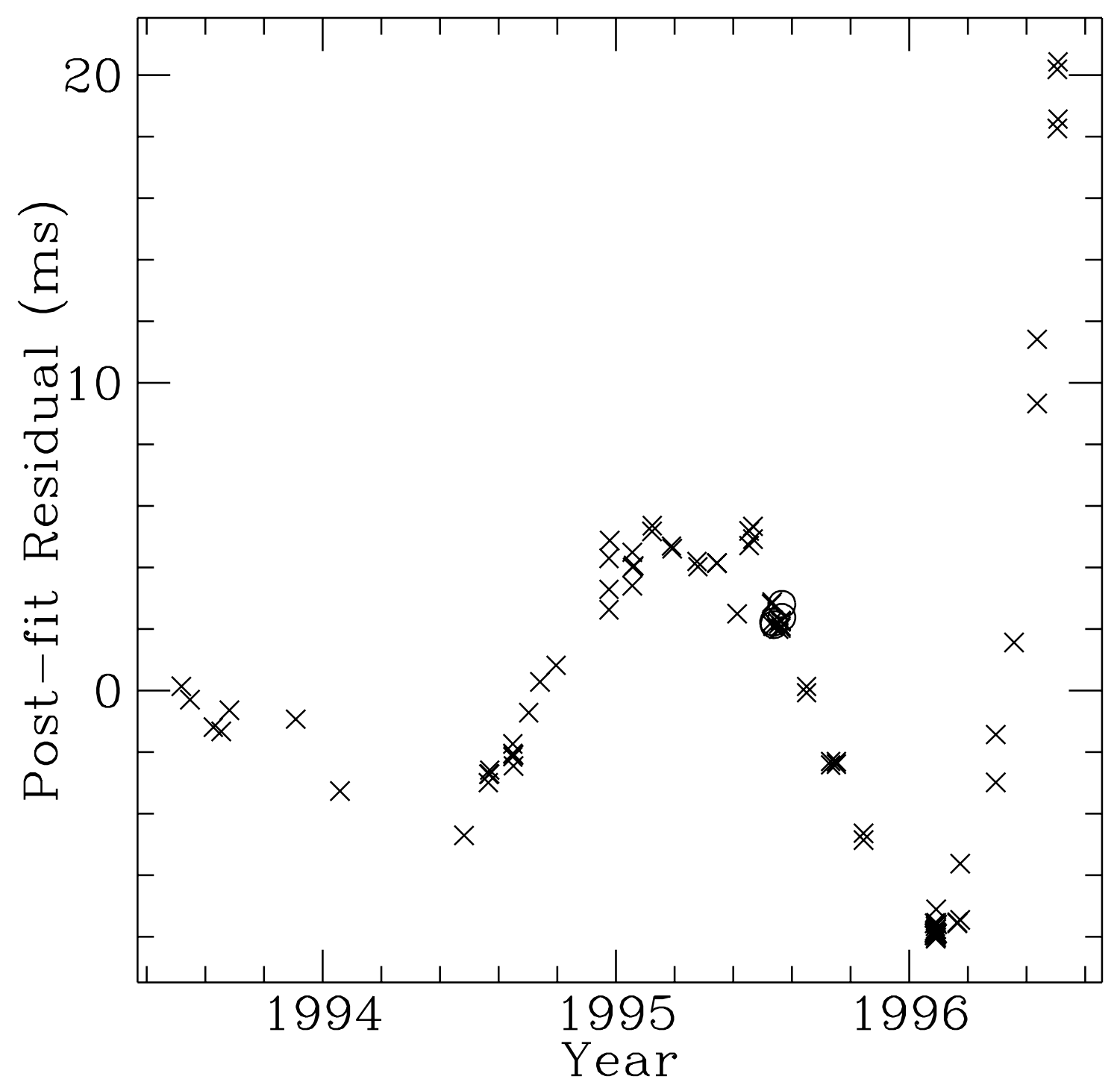

Fig. 2.- Post-fit residuals for PSR J1105-6107 after subtracting the model given in Table 1. Residuals at $660 \mathrm{MHz}$ are shown as open circles. Uncertainties on arrival times are typically smaller than the size of the symbols. 


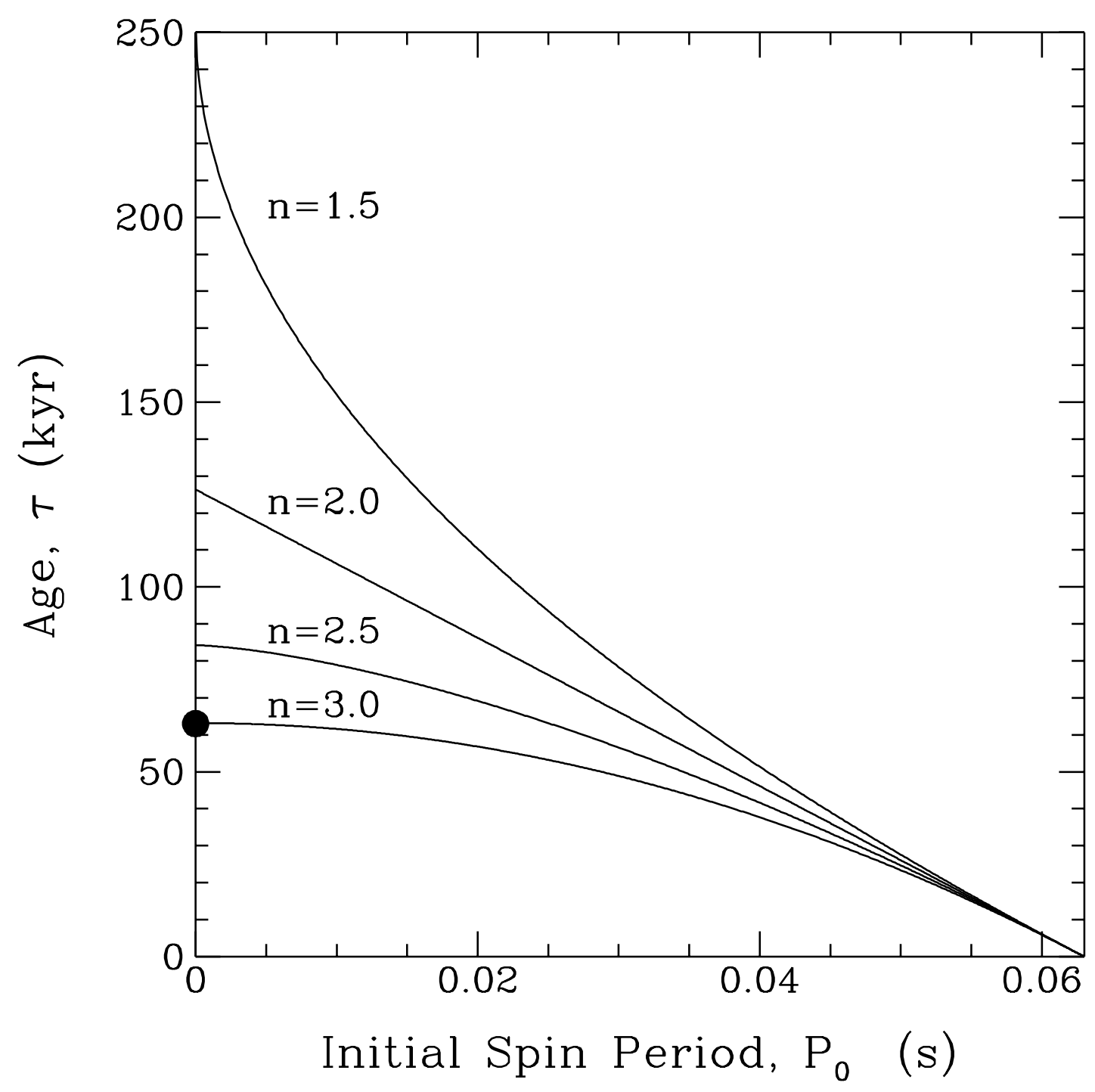

Fig. 3.- The true age $\tau$ of PSR J1105-6107 as a function of the assumed initial spin period $P_{0}$ for four values of the braking index $n$. The filled circle represents the characteristic age $\tau_{c} \equiv P / 2 \dot{P}$, typically assumed for a pulsar's age. 


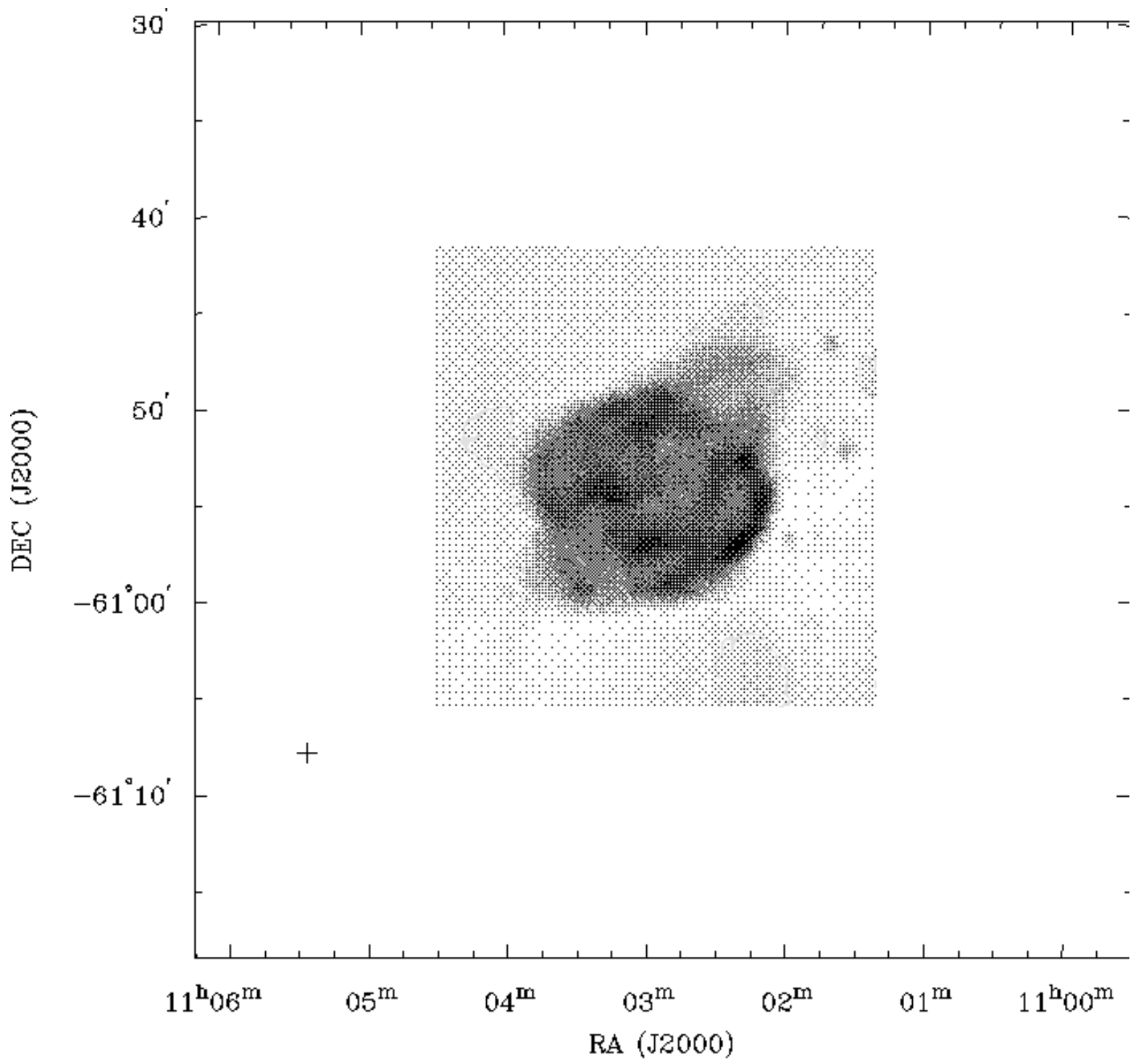

Fig. 4.- MOST radio image of G290.1-0.8 at $843 \mathrm{MHz}$ (after Whiteoak \& Green 1996). The position of the pulsar is indicated by a cross. 
Table 1. Astrometric, Spin, and Radio Parameters for PSR J1105-6107.

\begin{tabular}{|c|c|}
\hline Parameter & Value \\
\hline Right Ascension, $\alpha$ (J2000) & $11^{\mathrm{h}} 05^{\mathrm{m}} 26^{\mathrm{s}} .07(7)$ \\
\hline Declination, $\delta(\mathrm{J} 2000)$ & $-61^{\circ} \quad 07^{\prime} \quad 52^{\prime \prime} .1(4)$ \\
\hline Galactic Latitude, $l$ & $290^{\circ} .4896(2)$ \\
\hline Galactic Longitude, $b$ & $-0^{\circ} .8465(1)$ \\
\hline Period, $P$ & $0.063191252792(3) \mathrm{s}$ \\
\hline Period Derivative, $\dot{P}$ & $15.80466(12) \times 10^{-15}$ \\
\hline Dispersion Measure, DM & $271.01(2) \mathrm{pc} \mathrm{cm}^{-3}$ \\
\hline Epoch of Period & MJD 49545.0000 \\
\hline R.M.S. timing residual & $6.2 \mathrm{~ms}$ \\
\hline Surface Magnetic Field Strength, $B$ & $1.0 \times 10^{12} \mathrm{G}$ \\
\hline Characteristic Age, $\tau_{c}$ & $63,350 \mathrm{yr}$ \\
\hline Spin-Down Luminosity, $\dot{E}$ & $2.5 \times 10^{36} \mathrm{erg} \mathrm{s}^{-1}$ \\
\hline Flux Density at $660 \mathrm{MHz}^{1}$ & 4.1(7) mJy \\
\hline Flux Density at $1420 \mathrm{MHz}^{2}$ & $1.84(14) \mathrm{mJy}$ \\
\hline Flux Density at $1650 \mathrm{MHz}^{3}$ & $1.58(6) \mathrm{mJy}$ \\
\hline Spectral Index & $-1.36(1)$ \\
\hline $50 \%$ width at $1650 \mathrm{MHz}, \mathrm{W} 50$ & $3.4 \mathrm{~ms}=53.9 \mathrm{mP}$ \\
\hline $10 \%$ width at $1650 \mathrm{MHz}, \mathrm{W} 10$ & $4.8 \mathrm{~ms}=76.0 \mathrm{mP}$ \\
\hline
\end{tabular}

Note. - (1) reported flux is the mean of those measured at 3 epochs

(2) reported flux is the mean of that measured at 24 epochs

(3) reported flux is the mean of that measured at 16 epochs 